\title{
Medicina basada en la evidencia, origen, verdades, falacias y aceptación en Cuba
}

\author{
The origin, truths, fallacies of the evidence-based medicine and \\ its acceptance in Cuba
}

\author{
Dr. C. Rosa E. Jiménez Paneque \\ Hospital Clinicoquirúrgico "Hnos Ameijeiras". La Habana, Cuba.
}

\begin{abstract}
RESUMEN
La evidencia es conocimiento adquirido a través del método científico y este la herramienta principal que utiliza la ciencia en la búsqueda del conocimiento del mundo y de las causas de los fenómenos que ocurren en la realidad. Esta evidencia se difunde principalmente a través de la literatura científica, pero, muchos artículos originales provienen de estudios "con alto riesgo de sesgos" y se necesita una valoración crítica de los datos que aporta la literatura. La búsqueda de evidencia para la práctica médica no es un capricho sino la única vía para que el conocimiento verdadero tenga un impacto social real. La llamada Medicina basada en la evidencia, está basada en estos conceptos pero su seguimiento ortodoxo es prácticamente imposible e incosteable. Se reconoce no obstante, la existencia de un abismo entre lo que se demuestra como efectivo en la investigación y su aplicación práctica real en prescripciones médicas y sanitarias. A pesar de todo, la Medicina basada en la evidencia se ha expandido por casi todos los escenarios clínicos del mundo y ha trascendido hacia la salud pública y la política de salud. En Cuba, se aprecia interés en esta medicina en cuanto a la incorporación de nuevas evidencias científicas a la práctica médica. Los países en desarrollo, tienen la desventaja de contar con pocos estudios realizados en ambientes socioculturales propios. Se necesita promover la investigación de calidad, en los países no desarrollados que genere "evidencia propia" pues no es ético introducir prácticas solo basadas en conocimientos surgidos y demostrados en países desarrollados.
\end{abstract}

Palabras clave: Medicina basada en la evidencia, método científico, calidad de la investigación.

\section{ABSTRACT}

Evidence is knowledge acquired through the scientific method, being the latter the series of techniques and procedures that science uses in the search for knowledge 
about the world and the causes of phenomena. The evidence is primarily spread through scientific literature, but since many original articles come from studies "at high risk of bias", a critical appraisal of the data that the literature provides is needed. The search of scientific evidence for medical practice is not a whim, but the only way by which the scientific knowledge can produce real social impact. The so called Evidence Based Medicine (EBM) is supported on these concepts, but its orthodox practice is virtually impossible and unaffordable. However, the existence of a wide gap between what is demonstrated as effective through research and the actual implementation in medical and health prescriptions is admitted. Nevertheless, EBM has currently extended to almost all clinical scenarios worldwide and has transcended into public health and health policy. Some clear interest in the integration of new scientific evidence to medical practice in Cuba is noticeable. The developing countries face the disadvantage of little evidence originating from their own socio-cultural contexts. It is thus necessary to promote quality research in developing countries with the aim of generating their "own scientific evidence", since it is not ethical to bring in practices based only on knowledge acquired and demonstrated in developed countries.

Key words: Evidence based medicine, scientific method, research quality.

\section{NTRODUCCI ÓN}

La medicina clínica es la disciplina que se ocupa de la atención a personas enfermas y puede considerarse, al menos hoy, integrada por tres componentes básicos: 1. La experiencia y la habilidad clínica. 2. La evidencia científica existente sobre la eficacia y efectividad de los distintos procedimientos diagnósticos y terapéuticos. 3. Las preferencias expresas y las necesidades del paciente.

El primer componente es el más importante porque no existe hasta el momento manera de sustituir el razonamiento del médico, su conocimiento y habilidades, para conjugar lo que dice la evidencia científica, lo que dice su experiencia y lo que el paciente prefiere o necesita. Sin embargo, el componente que hemos denominado "evidencia científica" amerita atención especial, por sus variadas aristas y puntos controversiales y es el centro de este artículo.

Este binomio de palabras, alude a la evidencia como conocimiento adquirido a través del empleo del método científico en sentido general. El método científico se refiere al conjunto de procedimientos y técnicas de las que se vale la ciencia para sortear los obstáculos que suponen la subjetividad y las limitaciones físicas del hombre, para el conocimiento del mundo que le rodea y las causas de los fenómenos que ocurren en la realidad. El hombre mismo a través del tiempo ha concebido los métodos para conocer la realidad en la que vive y no por puro capricho o curiosidad (aunque algo de esto puede haber), sino porque solo conociéndola con certeza podrá transformarla según sus deseos y en el mejor de los casos transformarla para provecho del hombre mismo.

La evidencia científica, tal como ilustra la figura 1 , se adquiere principalmente a través de la literatura científica. Si bien la investigación es la manera principal de obtener conocimientos científicos, su divulgación para que puedan ser aplicados a la práctica social, se hace principalmente a través de las revistas científicas y dentro de 
estas, los artículos originales son los que dan cuenta de los resultados obtenidos en la búsqueda de respuestas a preguntas de investigación concretas.

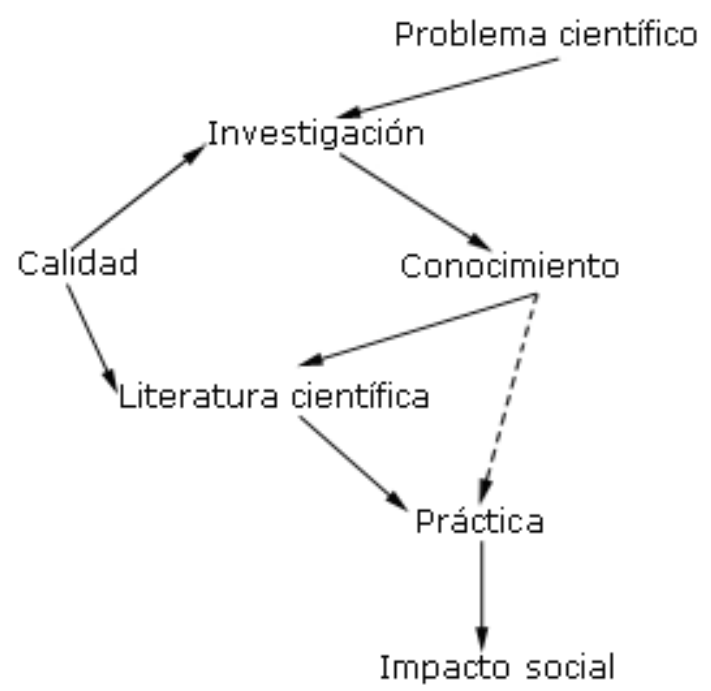

Fig. 1. Problema cientifico, calidad de la investigación e impacto social.

Pero, no toda la literatura científica, es portadora de conocimientos verdaderos porque, por razones diversas, se suelen publicar artículos originales de estudios con resultados claramente cuestionables o, como también se les dice, estudios "con alto riesgo de sesgos". Es obvio que estos resultados cuestionables no pueden producir el impacto esperado en la práctica social. Es en este sentido que se pide que, en los artículos originales, además de una proposición clara de las preguntas que se intenta responder en la investigación, se enuncie el método que se ha utilizado porque de él depende el alcance de la evidencia y las limitaciones de cada hallazgo.

El empleo de métodos apropiados para encontrar respuestas verdaderas a las preguntas es por tanto un punto crucial de la investigación primero y de la publicación de resultados después.

Por otro lado, un asunto ineludible y en íntima relación con el anterior, es que un estudio, realizado con solo una muestra de la realidad que se busca -ya que no es posible acceder a toda la realidad- no puede conducir a la verdad absoluta ya que, en biología al menos, la variabilidad de los seres vivos, entre los que se encuentra el hombre, impide que con solo una muestra se encuentre la respuesta inequívoca a determinada pregunta.

La estadística inferencial, con sus modelos de la realidad y procedimientos específicos, nos dice (dentro de límites aceptables) la probabilidad de equivocación en cada resultado encontrado en una muestra, con respecto a esa realidad que se estudia, muchas veces llamada "población objeto". Ese conocimiento es el que permite actuar pues una baja probabilidad implicará un riesgo bajo de cometer errores si se toman decisiones a partir de cierto resultado.

En resumen, si se emplea un diseño de investigación adecuado se habrá entrado en el camino de la verdad y los resultados serán verosímiles, si además se utilizan las herramientas de la estadística inferencial, se puede conocer el riesgo de posibles equivocaciones debidas a la inevitable necesidad de observar solo muestras. La 
certeza y sus circunstancias solo se alcanzan con el paso del tiempo, cuando esa verdad ocupe su lugar que, por demás, puede tener límites temporales y espaciales y relatividades diversas. ${ }^{1}$

Estos son en términos generales, los elementos que conforman la llamada evidencia científica, no se trata de un capricho de entendidos, sino la única forma en que el conocimiento verdadero pueda tener un impacto social real. ${ }^{2}$

Desconocer estos preceptos equivale a no entender que la desviación, bien sea por ignorancia o por desidia, del camino de la búsqueda eficaz de la verdad, es fuente potencial de catástrofes y en consecuencia origen de graves conflictos éticos.

\section{ORI GEN DE LA MEDICI NA BASADA EN LA EVI DENCIA}

En muchos sentidos, la práctica de la llamada Medicina Basada en la Evidencia (MBE) está relacionada con todos estos conceptos, algo que quizás no salta a la vista y que por tanto pocos destacan. Se desarrolla desde finales de los 80 y sobre todo en la década de los 90 y se deriva como un subproducto de la Epidemiología Clínica (EC), que se había desarrollado en la década anterior.

Alvan R Feinstein, padre de La EC entrega la siguiente definición de esta disciplina:

[...] la que representa la manera en la que la epidemiología clásica, tradicionalmente orientada hacia las estrategias en la salud pública de grupos comunitarios, se amplía para incluir las decisiones clínicas. En cuanto a métodos de investigación, la EC se ocupa de los procedimientos y standards necesarios para estudios científicamente rigurosos de los fenómenos clínicos complejos que ocurren en las personas. ${ }^{3}$

En la década de los 80 se publican también varios libros de texto dedicados a la Epidemiología Clínica. ${ }^{3-5}$ Uno de los libros principales y más conocidos sobre esta disciplina tiene como autor a David L Sackett, quien sería luego uno de los fundadores de la MBE. ${ }^{5}$

Además del ensayo clínico, diseño paradigmático de la investigación clínica para evaluar eficacia terapéutica, entre los principales métodos de la EC se encuentran también los que permiten evaluar la utilidad de medios diagnósticos y los que permiten probar hipótesis sobre potenciales factores pronóstico.

El desarrollo de la EC vino aparejado con la apertura de centros para su enseñanza en los países desarrollados; muchos médicos interesados en la nueva disciplina tuvieron la oportunidad de aprender a investigar en el mismo medio de la clínica y entender la aplicación del método científico para obtener conclusiones no cuestionables.

Aprendieron también a distinguir la literatura con resultados confiables de la poco confiable obtenida con métodos poco rigurosos.

Pero, en algún momento se hizo patente que, si bien los métodos de investigación en epidemiología habían migrado a la clínica, muchos resultados de la investigación se comenzaron a publicar en revistas importantes y las revistas comenzaron a solicitar artículos que se basaban en estudios realizados con el rigor que imponían los nuevos tiempos, la práctica de la medicina se mantenía atrasada.

En este contexto, Guyatt, Sackett y otros fundaron en la Universidad de McMaster en Ontario, Canadá, un grupo que tenía el objetivo de impulsar el desarrollo de lo que 
llamaron MBE. Sus motivos eran, o parecían bien razonables: si cada vez más, a través de la aplicación de los métodos de la EC surgían medios diagnósticos, procedimientos terapéuticos o conocimiento sobre determinados factores pronóstico que de alguna manera sugerían cambios en la conducta con el paciente, ¿por qué en la práctica médica se avanzaba poco en el empleo de tales métodos?, ¿por qué se continuaban indicando terapéuticas o medios diagnósticos menos eficaces? La respuesta la dieron varios estudios publicados en la época. Una encuesta realizada a un grupo de médicos generales en los EE. UU. encuentra que la mayoría de los médicos participantes leía menos de una hora a la semana sobre sus pacientes. ${ }^{6}$ En 1991 otro estudio revela que el conocimiento de los médicos sobre la mejor forma de atención a pacientes con hipertensión arterial (HTA) disminuye con el tiempo de graduados, lo que llamaron la "pendiente resbaladiza". ${ }^{7}$

Estos y otros elementos los condujeron a proponer un nuevo enfoque para la práctica de la medicina y le llamaron MBE. ${ }^{8,9}$ ¿En qué consistía este nuevo enfoque? La primera y quizás más certera respuesta la dan Rosenberg y Donald en 1995 cuando afirman que:

La MBE es un proceso que transforma los problemas clínicos en preguntas y después, de forma sistemática, localiza, recupera y utiliza los hallazgos de investigación más actuales como base para la toma de decisiones en la clínica. ${ }^{10}$

En concreto, la práctica de la MBE implica llevar adelante cuatro pasos (Fig.2).

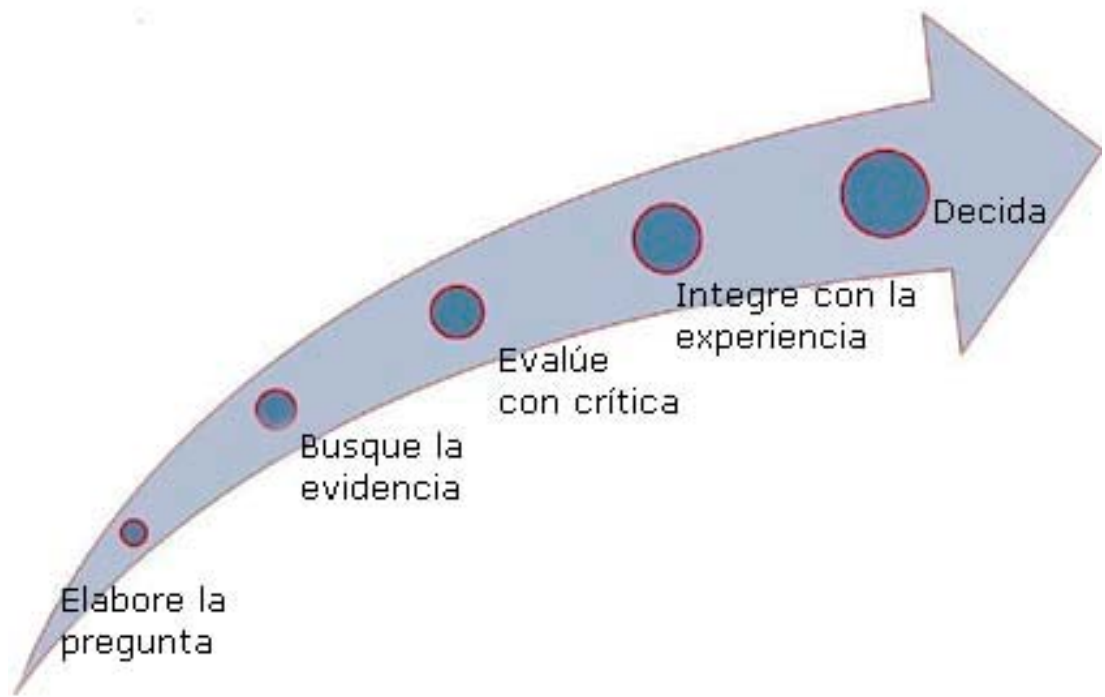

Fig. 2. Pasos para la práctica de la Medicina basada en la evidencia.

El primer paso es el de hacer las preguntas, es decir que, si bien el médico se ha hecho siempre preguntas ante un paciente durante todo el proceso diagnóstico cuyas respuestas guiaban este proceso inicial antes de tener elementos suficientes para prescribir un tratamiento, ahora la pregunta no va dirigida a pensar en la indicación de cierta investigación complementaria, según su conocimiento y experiencia, sino va a la búsqueda de la evidencia en la literatura, no es lo mismo. ${ }^{10}$ Esto implica que, además de aprender a hacer preguntas que conduzcan a respuestas claras dentro de la literatura existente, deben saber realizar búsquedas eficientes en las bases de datos bibliográficas y distinguir la buena literatura científica de la que tiene alto riesgo 
de sesgos. Una vez encontrada la evidencia adecuada, se podrá tomar una decisión en base a esta. De modo que, normalmente cada caso clínico tendría que pasar al menos dos veces por este camino, primero para diagnosticar y después para tratar. Con el fin de agilizar este largo proceso de preguntas y búsquedas se crearon herramientas. Entre estas se cuentan, en primer lugar, las revisiones sistemáticas de la literatura, estudios dirigidos a la búsqueda exhaustiva de artículos con respuesta potencial a la pregunta en cada caso y eventualmente acompañadas de métodos para resumir la información en esos artículos. ${ }^{11,12}$ Otras herramientas son las revistas que publican resúmenes filtrados de los resultados de las investigaciones en diversos temas y circunstancias. ${ }^{13,14}$ Se introdujeron los llamados niveles de evidencia según los cuales cada tipo de resultado brindaba más o menos evidencia de acuerdo al tipo de diseño empleado para su obtención y promovieron además la confección de las llamadas "guías de práctica clínica basadas en la evidencia" donde cada paso en la atención a determinada dolencia o afección debe estar respaldado por cierto nivel de evidencia en consonancia con la forma en que se ha llegado a obtener cada conocimiento. ${ }^{15,16}$

\section{FALACI AS Y PRÁCTI CA DE LA MEDI CI NA BASADA EN LA EVI DENCI A}

Con todas estas herramientas se suponía que, a pesar de lo engorroso que resulta seguir los pasos indicados y que mencionamos antes, la manera de practicar la medicina cambiaría radicalmente.

Pero, ¿realmente fue así? A todas luces, no. Los promotores de la MBE en principio no lograron la aceptación que esperaban o querían.

En primer lugar, las críticas empezaron rápidamente a llegar. En segundo lugar, es bastante claro que la práctica ortodoxa de la MBE tal como se dice en los libros de MBE originales, es prácticamente imposible. Totalmente imposible para marcos de urgencia por ejemplo, y en otros escenarios como los pases de visita en sala, también prácticamente imposible. Esta práctica en un escenario clínico demandaría un equipo enorme de personas auxiliares para realizar en cada paciente el ritual prescrito, primero para el diagnóstico y luego para el tratamiento. En realidad no sé cómo se les pudo ocurrir tal desatino que además de engorroso resultaría incosteable.

La MBE desde el principio recibió críticas muy agudas. Tannenbaum, por ejemplo señala que la MBE promovía la práctica médica basada sobre la evidencia científica obtenida en escenarios externos. ${ }^{16}$ Otros consideraban que la MBE era autoritaria y que promovía un libro de recetas. ${ }^{17}$ Muchos comentaron que no era un nuevo paradigma ¿cómo desconocer el hecho de que la medicina siempre se ha basado en el conocimiento científico? Las críticas en algunos casos fueron realmente severas, por ejemplo, Holmes y otros opinan que "constituye un buen ejemplo de microfascismo en la arena científica contemporánea."18

A raíz de estos comentarios, en 1995 Sackett publica un editorial en la BMJ, uno de los artículos más leídos sobre la MBE, cuyo sugestivo título dice: "MBE: ¿qué es y qué no es?"19 Aparece entonces la definición, ahora más extendida, con la cual intentan acallar una de las principales críticas dirigidas a la MBE y refieren que:

La MBE es el uso consciente, explícito y juicioso de la mejor evidencia actual para tomar decisiones sobre el cuidado de pacientes individuales.

En este mismo artículo señalan con tino que: 
Los buenos médicos utilizan tanto su experiencia clínica individual como la mejor evidencia externa y ninguna de las dos aisladamente es suficiente y añaden que, sin la experiencia clínica, la práctica médica corre el riesgo de ser tiranizada por la evidencia y que sin la mejor evidencia actual, la práctica médica corre el riesgo de perder actualidad rápidamente en detrimento de los pacientes.

Desde ese momento los promotores principales de la MBE defendieron estos conceptos lo que quizás les abrió el camino que ellos mismos habían cerrado con sus definiciones y proposiciones dogmáticas e impracticables.

Por último, el hecho de que la MBE haya surgido en un país industrializado donde las compañías farmacéuticas, casi siempre transnacionales, se erigen entre las más acaudaladas, levanta sospechas sobre un nexo, probablemente indirecto pero dañino, entre práctica de MBE e intereses comerciales. ${ }^{20,21}$

Ahora bien ¿cómo se ha practicado y se practica la MBE actualmente?

En 1996 y 1997 las revistas ACP J ournal Club y Evidence based Medicine, publicaron una serie de cuatro artículos destinados a describir cómo transferir la evidencia que producía la investigación a la práctica. ${ }^{22-25}$ Hacían hincapié en la importancia de evaluar la calidad de los artículos publicados y la forma de hacerlo. Pero, la respuesta a cómo se implementa la parafernalia de cosas que dice necesitar la práctica de la MBE no estaba respondida en estos artículos.

Sin embargo, en 1998 Sackett y otros evalúan la factibilidad de la aplicación de la evidencia a las decisiones clínicas durante los pases de visita. ${ }^{26}$ Describen un dispositivo al que llamaron "carrito de evidencia" supuestamente equipado con herramientas suficientes para el ritual de la práctica "evidencista". Los autores resumen que: la evidencia al estar disponible rápidamente para los clínicos en una sala de hospitalización con gran ocupación, mediante un carrito, aumenta el grado de búsqueda de evidencia y su incorporación a las decisiones clínicas, pero también señalan una serie de limitaciones para este estudio, amén de las complicaciones logísticas que conlleva tener un carrito, equiparlo y utilizarlo durante el pase de visita. ${ }^{26}$

No obstante, existe documentación suficiente sobre la existencia de un gran abismo entre lo que se demuestra como eficaz y efectivo en la investigación y su aplicación práctica real que, en buena ley, implicaría cambios en las prescripciones médicas y sanitarias. ${ }^{27}$ Como regla general se supone que la mejoría en la eficiencia del traspaso de los nuevos conocimientos científicos a la práctica, se logra a través de: mejor disposición y presentación de la evidencia mediante la identificación, síntesis y diseminación de esta a los médicos en formatos prácticos y accesibles, como por ejemplo las revisiones en revistas clínicas, guías de práctica clínica, mejor acceso a las fuentes electrónicas de información, educación médica continuada, cursos y conferencias. ${ }^{28}$ Pero, al igual que los avances en el tratamiento de la HTA no repercuten en una disminución de las consecuencias de esta enfermedad si no se logra que los enfermos conozcan que lo son, busquen atención médica y se adhieran al tratamiento; es necesario que los proveedores de salud sientan la necesidad de esta evidencia, la busquen, la entiendan y la apliquen. La preocupación por el abismo entre evidencia científica y práctica médica ha suscitado la publicación de propuestas diversas para disminuirlo. ${ }^{25-31}$

A pesar de todo, la MBE se ha difundido hoy por casi todos los escenarios clínicos del mundo y ha trascendido hacia la salud pública y la política de salud. ${ }^{32}$ 


\section{ACEPTACIÓN EN CUBA}

La incursión documentada de los profesionales de la medicina cubanos en la MBE es relativamente pobre y reciente. El artículo más antiguo que trata directamente el tema es de 2002 y fue publicado en la Revista Cubana de Hematología Inmunología y Hemoterapia, pretende dar un resumen de lo que hasta ese momento se conocía de la MBE y su forma de accionar. Se intenta con este artículo dar a conocer el concepto y los objetivos del movimiento MBE a los lectores de la revista y, aunque señalan algunas de sus limitaciones, se muestran más bien proclives a su introducción al calificarla como "proceso útil". ${ }^{33}$ En 2003, la Revista Cubana de Cirugía, publica un artículo sobre la MBE. La tónica del artículo refleja un respaldo claro de estos autores a la incorporación de la MBE a la práctica de la medicina. ${ }^{34}$ En 2005 Moreno establece un parangón entre la MBE y lo que llama la práctica médica individual, su leit motiv es que la atención médica es mucho más que los resultados de investigaciones realizadas a menudo en condiciones semideales aunque reconoce que las principales autoridades exponentes de la MBE no niegan la importancia de la experiencia personal de los médicos para la toma de decisiones con sus pacientes. ${ }^{35}$

En 2005, Vidal Ledo y Fernández Sacasas publican sus reflexiones sobre MBE en la revista de Educación Médica Superior, se refieren también a las controversias que existen entre el uso de la evidencia científica y la experiencia en la práctica médica individual. Concluyen aceptando la importancia de la MBE siempre que se integre a lo que llaman "competencia clínica personal". ${ }^{36}$ En 2008 se publica un artículo que, en el contexto de la MBE, se refiere a la importancia de la búsqueda de evidencia científica, para los métodos que promueve la llamada medicina alternativa o complementaria. ${ }^{37^{\prime}}$ Más recientemente, en 2011 Fernández Sacasas se refiere directamente a las controversias que existen en torno a la MBE, señala lo que llama pros y contras de la MBE y el peligro de que, con sus inmensas fortunas, sean las compañías farmacéuticas las que dirijan la evidencia y su búsqueda. No obstante, termina reconociendo la necesidad de la integración de la experiencia clínica con las evidencias científicas disponibles. ${ }^{38}$

Habiendo llegado hasta aquí, corresponde preguntarnos qué posibilidades y potenciales implicaciones podría tener la promoción de la práctica de la MBE en Cuba y si puede ser esto beneficioso o no.

La respuesta quizás transita por lo que se ha estado escribiendo sobre la práctica de la MBE en países no desarrollados. Sí, porque el movimiento de la MBE surgió en Canadá y, como era de esperar, la introducción de una u otra manera en la práctica de la medicina en general de los preceptos formulados por los promotores de esta corriente ha tenido lugar en países desarrollados.

En 2005 se publica un artículo dirigido especialmente a evaluar lo que puede ofrecer la MBE a los países no desarrollados. Señalan sus autores que la mayor carga de enfermedad en el mundo se encuentra en estos países y se parte de que en situaciones de bajos recursos, como es el caso, es entonces aun más necesario aplicar métodos de prevención o intervención que sean más eficaces y efectivos. Comentan tres circunstancias que limitan el empleo de la MBE en países no desarrollados: las revisiones sistemáticas existentes no reflejan las prioridades del mundo no desarrollado, muchas intervenciones respaldadas por estas revisiones no pueden implementarse en situaciones de bajos recursos, y la investigación primaria se conduce poco en países no desarrollados. Reconocen que en países no desarrollados no basta con la intención de aplicar intervenciones de salud que han probado ser efectivas, sino que se necesita el concurso de otras esferas de la sociedad y cambios en las políticas sociales de modo que abogan por aumentar las revisiones sistemáticas 
que aborden los problemas de esos países y se basen en investigación realizada en ellos. $^{39}$

También en 2005 se publica una revisión sistemática sobre la efectividad de intervenciones dirigidas a cambiar la conducta de los profesionales de la salud en relación con la incorporación de la evidencia a sus prácticas en países no desarrollados. Encuentran 44 estudios que cumplen con sus criterios de inclusión y que la mayoría muestra eficacia en la intervención explorada, pero los autores temen por un importante sesgo de publicación en este caso, donde la literatura proviene de países no desarrollados. Así mismo, señalan que la mayoría de los estudios tienen un pobre diseño metodológico. Recomiendan que las instituciones internacionales con experiencia en sistemas de salud provean ayuda técnica a los países en desarrollo para la realización de estudios apropiados. ${ }^{40}$

\section{CONSI DERACI ONES FI NALES}

A pesar de las muchas críticas recibidas con mayor o menor fundamento por la MBE, esta constituyó un llamado claro a la necesidad de investigación convincente e introducción efectiva de los nuevos conocimientos en la práctica de la medicina. Su extensión a todos los ámbitos de las ciencias médicas y a la mayoría de los países es una expresión de la acogida favorable que ha tenido por parte de los profesionales de la salud en general. No es posible que en pleno siglo XXI los conocimientos encontrados a través de la investigación científica no contribuyan eficientemente a mejorar la vida del hombre. Por otro lado, si bien la MBE nació y se desarrolló en países desarrollados y con recursos, la aplicación en la práctica de procedimientos más eficaces y efectivos no puede sino redundar, a mediano y largo plazo, en una disminución de los costos de la asistencia médica, algo particularmente necesario en países en desarrollo; una verdadera paradoja que contribuye a perpetuar la diferencia entre países ricos y pobres.

El reto entonces para los países no desarrollados y de Cuba en particular, es introducir estos preceptos a favor de la población sin exclusiones porque la salud, como hemos defendido durante más de 50 años, es un derecho de todos.

Otra actitud sería una burla a los preceptos éticos y morales que preservamos. Se necesita también promover la investigación de calidad en los países no desarrollados para que contribuyan a lo que podemos llamar la "evidencia propia" pues tampoco sería ético introducir prácticas basadas en conocimientos surgidos y demostrados en ambientes socioculturales diferentes.

\section{REFERENCI AS BI BLI OGRÁFICAS}

1. Jiménez RE. Problema científico, población y muestra. Rev Cubana Cardiol Cir Cardiovasc. 2010;16(2):206-13.

2. Gutiérrez AR, Jiménez RE. ¿Por qué y cómo evaluar la calidad de la investigación científica en medicina? Rev Cubana Cardiol Cir Cardiovasc. 2011;17(4):365-70.

3. Feinstein AR. Clinical epidemiology: the architecture of clinical research. Philadelphia: Saunders; 1985. 
4. Fletcher RH, Fletcher SW, Wagner EH. Clinical Epidemiology. The essentials. 2sd ed. Baltimore: Williams \& Wilkins; 1988.

5. Sackett DL, Haynes RB, Tugwell P. Clinical Epidemiology; A Basic Science for Clinical Medicine. Boston: Little, Brown; 1985.

6. Covell DG, Uman GC, Manning PR. Information needs in office practice: Are they being met? Ann Intem Med. 1985;103(4):596-9.

7. Ramsey PG, Carline JD, Inui TS, Larson EB, LoGerfo JP, Norcini JJ, et al. Changes over time in the knowledge base of practicing internists. JAMA. 1991;266:1103-7.

8. Evidence-Based Medicine Working Group. Evidence-Based Medicine A New Approach to Teaching the Practice of Medicine. JAMA. 1992;268:2420-5.

9. David L Sackett, William M C Rosenberg. The need for evidence-based medicine. J R Soc Med. 1995;88:620-4.

10. Rosenberg W, Donald A. Evidence based medicine: an approach to clinical problem-solving. BMJ. 1995;310(6987):1122-6.

11. Cook DJ, Mulrow CD, Haynes RB. Systematic reviews: synthesis of best evidence for clinical practice. Ann Intern Med. 1997;126:37680.

12. López Arrieta JM, Qizilbash N. Evidence-based medicine: systematic reviews. The Cochrane Collaboration. Med Clin (Barc). 1996;107(15):581-5.

13. Davidoff F, Haynes B, Sackett DL, Smith R.Evidence based medicine. A new journal to help doctors identify the information they need [letter]. BMJ. $1995 ; 310: 1085-86$.

14. Woolf SH, Battista RN, Anderson GM, Logan AG, Wang E, Goldbloom RB, et al. Assessing the clinical effectivenes of preventive maneuvers: analytic principles and systematic methods in reviewing evidence and developing clinical practice recommendations. J Clin Epidemiol. 1990;43:891-905.

15. Browman G, Gómez de la Cámara A, Haynes B, Jadad A, Gabriel R. Herramientas para la práctica de la medicina basada en la evidencia. Desarrollo de guías de práctica clínica basadas en la evidencia: de abajo-arriba. Med Clin (Barc). 2001;116:267-70.

16. Tannenbaum S. Getting there from here: evidentiary quandaries of the US outcomes movement. J Eval Clin Pract. 1995;1:97-103.

17. Charlton BG. Restoring the Balance: Evidence-basedMedicine Put in Its Place. J Evaluation Clin Pract. 1997;3(2):8798.

18. Holmes D, Murray SJ, Perron A, Rail G. Deconstructing the evidence-based discourse in health sciences: truth, power and fascism. Int J Evid Based Health. $2006 ; 4: 1806$.

19. Evidence based medicine: what it is and what it isn't [editorial].

BMJ. 1996;312:71.

http://scielo.sld.cu 
20. Abramson J, Starfield B. The Effect of Conflict of Interest on Biomedical Research and Clinical Practice Guidelines: Can We Trust the Evidence in Evidence-Based Medicine? JABFP. 2005;18(5):414-8.

21. Brennan TA, Rothman DJ, Blank L, Blumenthal D, Chimonas SC, Cohen JJ, et al. Health Industry Practices That Create Conflicts of Interest. A Policy Proposal for Academic Medical Centers. JAMA. 2006;295(4):429-33.

22. Haynes RB, Sackett DL, Gray JM, Cook DJ, Guyatt GH. Transferring evidence from research into practice: The role of clinical care research evidence in clinical decisions. ACP J Club. 1996;125(3):A14-6.

23. Haynes RB, Sackett DL, Gray JA, Cook DL, Guyatt GH.

Transferring evidence from research into practice: Getting the evidence straight. ACP J Club. 1997;126(1):A14-6.

24. Muir Gray JA, Haynes RB, Sackett DL, Cook DJ, Guyatt GH.

Transferring evidence from research into practice: Developing evidence-based clinical policy. ACP J Club. 1997;126(2):A14-6.

25. Haynes RB, Sackett DL, Guyatt GH, Cook DJ, Gray JA.

Transferring evidence from research into practice: 4 . Overcoming barriers to application. ACP J Club. 1997;126(3):A14-5.

26. Sackett DL, Straus SE. Finding and Applying Evidence During Clinical Rounds. The "Evidence Cart". JAMA. 1998;280(15):1336-8.

27. Lenfant C. Clinical research to clinical practice. Lost in translation? N Engl J Med. 2003;349(9):868-74.

28. Grol R, Grimshaw]. From best evidence to best practice: effective implementation of change in patients' care. Lancet. 2003;362:122530.

29. Wilton NK, Slim AM. Application of the Principles of Evidence-Based Medicine to Patient Care. Southern Med J. 2012;105(3):136-43.

30. Grol R. Beliefs and evidence in changing clinical practice. BMJ. 1997;315:41821.

31. Rosser W. Bringing important research evidence into practice: Canadian developments. Family Prac. 2008;25:13843.

32. Satterfield JM, Spring B, Brownson RC, Mullen EJ, Newhouse RP, Walker BB, et al. Toward a Transdisciplinary Model of Evidence-Based Practice. Milbank Q. 2009;87(2):368-90.

33. Lam Díaz RM, Oliva M, Hernández P, Milanés MT. Medicina basada en la evidencia. Rev Cubana Hematol Inmunol Hemoter [Internet]. 2002 [citado 8 Ago 2012];18(3). Disponible en: http://scielo.sld.cu/scielo.php?script=sci arttext\&pid=S0864$\underline{02892002000300002 \& \operatorname{lng}=\mathrm{es}}$

34. Adefna RI, Leal A, Izquierdo FT, Castellanos JA, Arocha R. De la "Medicina basada en la opinión" a la "Medicina basada en la evidencia". Rev Cubana Cir [Internet]. 2003 [citado 8 Ago 2012];42(1). Disponible en:

http://scielo.sld.cu/scielo.php?script=sci arttext\&pid=S003474932003000100008\&lng=es

http://scielo.sld.cu 
35. Moreno MA. La medicina basada en la evidencia y la práctica médica Individual. Rev Cubana Med [Internet]. 2005 [citado 8 Ago 2012];44(3-4). Disponible en: http://scielo.sld.cu/scielo.php?script=sci arttext\&pid=S003475232005000400015\&lng=es

36. Vidal M, Fernández JA. Medicina basada en la evidencia. Reflexiones sobre el tema. Educ Med Super [Internet]. 2005 [citado 8 Ago 2012];19(1). Disponible en: http://scielo.sld.cu/scielo.php?script=sci arttext\&pid=S0864$\underline{21412005000100010 \& \operatorname{lng}=\mathrm{es}}$

37. Morón F. Evidencia y uso de plantas medicinales en los sistemas de salud [editorial]. Rev Cubana Plant Med [Internet]. 2008 [citado 8 Ago 2012];13(1). Disponible en: http://scielo.sld.cu/scielo.php?script=sci arttext\&pid=S1028$\underline{47962008000100001 \& \operatorname{lng}=\mathrm{es}}$

38. Fernández JA. Controversias en torno a la medicina basada en evidencias. Rev Habanera Ciencias Médicas [Internet]. 2011 [citado Ago 2012 ];10(3):339-47. Disponible en: http://scielo.sld.cu/scielo.php?script=sci arttext\&pid=S1729$\underline{519 \times 2011000300012 \& \operatorname{lng}=\mathrm{es}}$

39. McMichael C, Waters E, Volmink J. Evidence-based public health: what does it offer developing countries? J Public Health. 2005;27(2):215-21.

40. Siddiqi K, Newell J, Robinson M. Getting evidence into practice: what works in developing countries? Internat J Quality Health Care. 2005; 17(5):44753.

Rosa E. Jiménez Paneque. Hospital Clinicoquirúrgico "Hermanos Ameijeiras". San Lázaro 701, Centro Habana. La Habana, Cuba.

Correo electrónico: rjimenez@infomed.sld.cu 